Volume 131, Number 3, Pages 815-823

S 0002-9939(02)06564-4

Article electronically published on July 2, 2002

\title{
SEGAL-BARGMANN TRANSFORMS OF ONE-MODE INTERACTING FOCK SPACES ASSOCIATED WITH GAUSSIAN AND POISSON MEASURES
}

\author{
NOBUHIRO ASAI, IZUMI KUBO, AND HUI-HSIUNG KUO \\ (Communicated by Claudia M. Neuhauser)
}

\begin{abstract}
Let $\mu_{g}$ and $\mu_{p}$ denote the Gaussian and Poisson measures on $\mathbb{R}$, respectively. We show that there exists a unique measure $\tilde{\mu}_{g}$ on $\mathbb{C}$ such that under the Segal-Bargmann transform $S_{\mu_{g}}$ the space $L^{2}\left(\mathbb{R}, \mu_{g}\right)$ is isomorphic to the space $\mathcal{H} L^{2}\left(\mathbb{C}, \widetilde{\mu}_{g}\right)$ of analytic $L^{2}$-functions on $\mathbb{C}$ with respect to $\widetilde{\mu}_{g}$. We also introduce the Segal-Bargmann transform $S_{\mu_{p}}$ for the Poisson measure $\mu_{p}$ and prove the corresponding result. As a consequence, when $\mu_{g}$ and $\mu_{p}$ have the same variance, $L^{2}\left(\mathbb{R}, \mu_{g}\right)$ and $L^{2}\left(\mathbb{R}, \mu_{p}\right)$ are isomorphic to the same space $\mathcal{H} L^{2}\left(\mathbb{C}, \widetilde{\mu}_{g}\right)$ under the $S_{\mu_{g}}$ and $S_{\mu_{p}}$-transforms, respectively. However, we show that the multiplication operators by $x$ on $L^{2}\left(\mathbb{R}, \mu_{g}\right)$ and on $L^{2}\left(\mathbb{R}, \mu_{p}\right)$ act quite differently on $\mathcal{H} L^{2}\left(\mathbb{C}, \widetilde{\mu}_{g}\right)$.
\end{abstract}

\section{IntRoduCtion}

Let $\mu$ be a probability measure on $\mathbb{R}$ having finite moments of all orders. In the paper [1] Accardi and Bożejko discovered a canonical unitary isomorphism between the Hilbert space $L^{2}(\mu)$ and the one-mode interacting Fock space $\Gamma(\lambda)$ associated with a sequence $\lambda$ arising from $\mu$. Under this isomorphism the number vectors $\Phi_{n}, n \geq 0$, correspond to the orthogonal polynomials $P_{n}(x)$ associated with $\mu$ and the modified field operator on $\Gamma(\lambda)$ corresponds to the multiplication operator by $x$ on $L^{2}(\mu)$.

Being motivated by Accardi-Bożejko's discovery, Asai has recently introduced in [3] coherent vectors which are used to define the $S_{\mu}$-transform. The $S_{\mu}$-transform is shown in $\left[3\right.$ to be a unitary operator from $L^{2}(\mu)$ onto a Hilbert space $\mathcal{H}_{\lambda}$ of analytic functions on a disk $\Omega_{\lambda} \subset \mathbb{C}$, where $\lambda$ is determined by $\mu$. The composition of the Accardi-Bożejko isomorphism and the $S_{\mu}$-transform gives the interacting Fock space counterpart of the well-known Segal-Bargmann transform (cf. [9, 10, 17, 22, 23).

The purpose of this paper is to apply the results of Accardi-Bożejko [1] and Asai [3] to the cases of Gaussian measure $\mu_{g}$ and Poisson measure $\mu_{p}$. In paticular, when $\mu_{g}$ and $\mu_{p}$ have the same variance, we will see that by Theorems 4.6 and 4.8

Received by the editors August 18, 2001 and, in revised form, October 12, 2001.

2000 Mathematics Subject Classification. Primary 46L53; Secondary 33D45, 44A15.

Key words and phrases. Interacting Fock space, Segal-Bargmann transform, coherent vector, Gaussian measure, Poisson measure, space of square integrable analytic functions, decomposition of multiplication operator.

Research of the first author supported by a Postdoctoral Fellowship of the International Institute for Advanced Studies, Kyoto, Japan. 
the Segal-Bargmann transforms $S_{\mu_{g}}$ and $S_{\mu_{p}}$ take $L^{2}\left(\mu_{g}\right)$ and $L^{2}\left(\mu_{p}\right)$, respectively, to the same space $\mathcal{H} L^{2}\left(\mathbb{C}, \widetilde{\mu}_{g}\right)$ of analytic $L^{2}$-functions, where $\widetilde{\mu}_{g}$ is the Gaussian measure on $\mathbb{C}$. However, the Segal-Bargmann representation of multiplication by $x$ on $L^{2}\left(\mu_{g}\right)$ is quite different from that on $L^{2}\left(\mu_{p}\right)$.

\section{One-mode interacting Fock SPaCe}

Let $\mu$ be a probability measure on $\mathbb{R}$ having finite moments of all orders. It is well known 25] that there exist (1) a complete orthogonal system $\left\{P_{n}(x)\right\}_{n=0}^{\infty}$ of polynomials for $L^{2}(\mu)$ with $P_{0}=1,(2)$ a sequence $\left\{\omega_{n}\right\}_{n=1}^{\infty}$ of nonnegative real numbers, and (3) a sequence $\left\{\alpha_{n}\right\}_{n=0}^{\infty}$ of real numbers such that the following equalities hold for all $n \geq 0$ :

$$
\begin{aligned}
\left(x-\alpha_{n}\right) P_{n}(x) & =P_{n+1}(x)+\omega_{n} P_{n-1}(x), \\
\left\langle P_{n}, P_{m}\right\rangle_{L^{2}(\mu)} & =\delta_{n, m} \omega_{1} \cdots \omega_{n},
\end{aligned}
$$

where $\omega_{0} P_{-1}=0$ by convention. We have the fact that the sequence $\alpha_{n}=0$ for all $n$ if and only if $\mu$ is symmetric.

For a probability measure $\mu$ with the associated sequence $\left\{\omega_{n}\right\}_{n=1}^{\infty}$, we define a sequence $\left\{\lambda_{n}\right\}_{n=0}^{\infty}$ by

$$
\lambda_{0}=1, \quad \lambda_{n}=\omega_{1} \omega_{2} \cdots \omega_{n}, \quad n \geq 1 .
$$

Assume that the sequence $\left\{\lambda_{n}\right\}_{n=0}^{\infty}$ satisfies the condition:

$$
\text { (*) } \quad \inf _{n \geq 0} \lambda_{n}^{\frac{1}{n}}>0 .
$$

With such a sequence $\lambda=\left\{\lambda_{n}\right\}_{n=0}^{\infty}$, we define $\Gamma(\lambda)$ by

$$
\Gamma(\lambda)=\left\{\left(a_{0}, a_{1}, \ldots, a_{n}, \ldots\right): a_{n} \in \mathbb{C}, \sum_{n=0}^{\infty} \lambda_{n}\left|a_{n}\right|^{2}<\infty\right\}
$$

and a norm $\|\cdot\|_{\lambda}$ on $\Gamma(\lambda)$ by

$$
\left\|\left(a_{n}\right)\right\|_{\lambda}=\left(\sum_{n=0}^{\infty} \lambda_{n}\left|a_{n}\right|^{2}\right)^{1 / 2} .
$$

Then $\Gamma(\lambda)$ is a Hilbert space with norm $\|\cdot\|_{\lambda}$. It is called the one-mode interacting Fock space associated with $\lambda[1,2]$.

Define a number vector $\Phi_{n}, n \geq 0$, by

$$
\Phi_{n}=(0, \ldots, 0, \stackrel{n+1}{1}, 0, \ldots) .
$$

The vector $\Phi_{0}$ is called a vacuum vector. Let $A$ be a densely defined operator on $\Gamma(\lambda)$ such that

$$
A \Phi_{0}=0, \quad A \Phi_{n}=\omega_{n} \Phi_{n-1}, \quad n \geq 1 .
$$

The adjoint operator $A^{*}$ of $A$ is easily checked to be given by

$$
A^{*} \Phi_{n}=\Phi_{n+1}, \quad n \geq 0 .
$$

The operators $A$ and $A^{*}$ are called the annihilation and creation operators on $\Gamma(\lambda)$, respectively. The number operator $N$ is defined by

$$
N \Phi_{n}=n \Phi_{n}, \quad n \geq 0 .
$$


In addition, we define an operator $\alpha_{N}$ on $\Gamma(\lambda)$ by

$$
\alpha_{N} \Phi_{n}=\alpha_{n} \Phi_{n}, \quad n \geq 0
$$

Now we can state the result of Accardi and Bożejko [1]: There exists a unitary isomorphism $U: \Gamma(\lambda) \rightarrow L^{2}(\mu)$ satisfying the following conditions:

(1) $U \Phi_{0}=1$

(2) $U A^{*} U^{*} P_{n}=P_{n+1}$

(3) $U\left(A+A^{*}+\alpha_{N}\right) U^{*}=Q$, where $Q$ is the multiplication operator by $x$ on $L^{2}(\mu)$.

\section{Segal-Bargmann transform}

Let $\left\{\lambda_{n}\right\}_{n=0}^{\infty}$ be the sequence defined in Equation (2.2) and consider the following series of a complex number $z$ :

$$
G_{\lambda}(z)=\sum_{n=0}^{\infty} \frac{1}{\lambda_{n}} z^{n}
$$

Note that by Condition $(\star)$ in Equation (2.3) this series has a positive radius of convergence, denoted by $r_{\lambda}$.

Let $\Omega_{\lambda}=\left\{z \in \mathbb{C}:|z|<\sqrt{r_{\lambda}}\right\}$. For each $z \in \Omega_{\lambda}$, Asai [3] has introduced a coherent vector $E_{\lambda}(\cdot, z)$ with respect to the family $\left\{P_{n}\right\}$ in Equation (2.1) by

$$
E_{\lambda}(x, z)=\sum_{n=0}^{\infty} \frac{P_{n}(x)}{\lambda_{n}} z^{n}, \quad x \in \mathbb{R} .
$$

It is easy to see

$$
\left\|E_{\lambda}(\cdot, z)\right\|_{L^{2}(\mu)}=G_{\lambda}\left(|z|^{2}\right)^{1 / 2}
$$

and so $E_{\lambda}(\cdot, z) \in L^{2}(\mu)$ for all $z \in \Omega_{\lambda}$. Moreover, the set $\left\{E_{\lambda}(\cdot, z): z \in \Omega_{\lambda}\right\}$ is linearly independent and spans a dense subspace of $L^{2}(\mu)$.

For $f \in L^{2}(\mu)$, let $S_{\mu} f$ be the function defined by:

$$
\left(S_{\mu} f\right)(z)=\left\langle E_{\lambda}(\cdot, \bar{z}), f\right\rangle_{L^{2}(\mu)}=\int_{\mathbb{R}} E_{\lambda}(x, z) f(x) d \mu(x), \quad z \in \Omega_{\lambda} .
$$

The mapping $S_{\mu}$ defined on $L^{2}(\mu)$ is called the Segal-Bargmann transform. Asai has shown in [3] that $S_{\mu}$ is a unitary operator from $L^{2}(\mu)$ onto $\mathcal{H}_{\lambda}$. Here $\mathcal{H}_{\lambda}$ is given by

$$
\mathcal{H}_{\lambda}=\left\{F(z)=\sum_{n=0}^{\infty} a_{n} z^{n}: F \text { is analytic on } \Omega_{\lambda} \text { and } \sum_{n=0}^{\infty} \lambda_{n}\left|a_{n}\right|^{2}<\infty\right\} .
$$

It is a Hilbert space with the norm

$$
\|F\|_{\mathcal{H}_{\lambda}}=\left(\sum_{n=0}^{\infty} \lambda_{n}\left|a_{n}\right|^{2}\right)^{1 / 2}
$$

Now, let us introduce operators $\widetilde{A}$ and $\widetilde{A}^{*}$ acting on $\mathcal{H}_{\lambda}$ by

$$
\widetilde{A} 1=0, \quad \widetilde{A} z^{n}=\omega_{n} z^{n-1}, \quad n \geq 1,
$$

and

$$
\widetilde{A}^{*} z^{n}=z^{n+1}, \quad n \geq 0 .
$$


Operators $\widetilde{A}$ and $\widetilde{A}^{*}$ satisfy the commutation relation $\left[\widetilde{A}, \widetilde{A}^{*}\right] z^{n}=\left(\omega_{n+1}-\omega_{n}\right) z^{n}$.

The number operator $\widetilde{N}$ acting on $\mathcal{H}_{\lambda}$ is defined by

$$
\widetilde{N} z^{n}=n z^{n}, \quad n \geq 0 .
$$

In addition, we can define an operator $\widetilde{\alpha}_{N}$ acting on $\mathcal{H}_{\lambda}$ by

$$
\widetilde{\alpha}_{N} z^{n}=\alpha_{n} z^{n}, \quad n \geq 0 .
$$

The operators $\widetilde{A}, \widetilde{A}^{*}, \widetilde{N}$ and $\widetilde{\alpha}_{N}$ correspond to the operators $A, A^{*}, N$ and $\alpha_{N}$ on $\Gamma(\lambda)$, respectively.

\section{MAIN RESUlts}

Our main results (Theorems 4.6 and 4.8 below) in this papers are concerned with the special cases when $\mu$ is a Gaussian or Poisson measure.

Case 1. $\mu=$ Gaussian measure $\mu_{g}$ with mean $m$ and variance $\sigma^{2}$.

From [19, 25] we have the following equalities:

$$
\begin{aligned}
& H_{n+1}\left(x ; \sigma^{2}\right)-x H_{n}\left(x ; \sigma^{2}\right)+\sigma^{2} n H_{n-1}\left(x ; \sigma^{2}\right)=0, \\
& \int_{\mathbb{R}} H_{n}\left(x ; \sigma^{2}\right) H_{m}\left(x ; \sigma^{2}\right) \frac{1}{\sqrt{2 \pi \sigma^{2}}} e^{-x^{2} / 2 \sigma^{2}} d x=\delta_{n, m} \sigma^{2 n} n !,
\end{aligned}
$$

where $H_{n}\left(x ; \sigma^{2}\right)$ is the Hermite polynomial of degree $n$ with parameter $\sigma^{2}$. Thus the three quantities for the Gaussian measure $\mu_{g}$ in Equation (2.1) are given by

$$
\begin{aligned}
P_{n}(x) & =H_{n}\left(x-m ; \sigma^{2}\right), \\
\omega_{n} & =\sigma^{2} n, \\
\alpha_{n} & =m \quad \forall n \geq 1 .
\end{aligned}
$$

Moreover, we have the associated quantities in Equations (2.2), (3.1), and (3.2):

$$
\begin{aligned}
\lambda_{n} & =\sigma^{2 n} n !, \\
G_{\lambda}(z) & =\exp \left[\frac{z}{\sigma^{2}}\right], \quad r_{\lambda}=\infty, \\
E_{\lambda}(x, z) & =\exp \left[\frac{z}{\sigma^{2}}(x-m)-\frac{z^{2}}{2 \sigma^{2}}\right] .
\end{aligned}
$$

Obviously, Condition ( $\star$ ) in Equation (2.3) is satisfied.

Case 2. $\mu$ = Poisson measure $\mu_{p}$ with parameter $a$.

From [11] we have the following equalities:

$$
\begin{aligned}
& C_{n+1}(x ; a)=(x-n-a) C_{n}(x ; a)-a n C_{n-1}(x ; a), \\
& \int_{\mathbb{R}} C_{n}(x ; a) C_{m}(x ; a) d \mu_{p}(x)=\delta_{n, m} a^{n} n !,
\end{aligned}
$$

where $C_{n}(x ; a)$ is the Charlier polynomial of degree $n$ with parameter $a$. Thus the three quantities for the Poisson measure $\mu_{p}$ in Equation (2.1) are given by

$$
\begin{aligned}
P_{n}(x) & =C_{n}(x ; a), \\
\omega_{n} & =a n, \\
\alpha_{n} & =n+a .
\end{aligned}
$$


Moreover, we have the associated quantities in Equations (2.2), (3.1), and (3.2):

$$
\begin{aligned}
\lambda_{n} & =a^{n} n ! \\
G_{\lambda}(z) & =\exp \left[\frac{z}{a}\right], \quad r_{\lambda}=\infty, \\
E_{\lambda}(x, z) & =e^{-z}\left(1+\frac{z}{a}\right)^{x} .
\end{aligned}
$$

Obviously, Condition ( $\star$ ) in Equation (2.3) is satisfied.

For the Poisson case, we have

$$
\alpha_{N}=\frac{1}{a} A^{*} A+a=N+a,
$$

which implies that

$$
A^{*}+A+\alpha_{N}=\left(\frac{1}{\sqrt{a}} A^{*}+\sqrt{a}\right)\left(\frac{1}{\sqrt{a}} A+\sqrt{a}\right) .
$$

Proposition 4.1. For the Gaussian measure $\mu_{g}$ on $\mathbb{R}$ with mean $m$ and variance $\sigma^{2}$, the following equalities hold:

(a) $S_{\mu_{g}} H_{n}\left(\cdot-m ; \sigma^{2}\right)=z^{n}$

(b) $S_{\mu_{g}} U A U^{*} H_{n}\left(\cdot-m ; \sigma^{2}\right)=\sigma^{2} n z^{n-1}$,

(c) $S_{\mu_{g}} U A^{*} U^{*} H_{n}\left(\cdot-m ; \sigma^{2}\right)=z^{n+1}$,

(d) $S_{\mu_{g}}\left((x-m) H_{n}\left(\cdot-m ; \sigma^{2}\right)\right)=z^{n+1}+\sigma^{2} n z^{n-1}$.

Proof. Conclusion (a) follows from Equations (3.2), (3.3), 4.1), and (4.2). (b) follows from (a) and the fact that $U A U^{*} H_{n}\left(\cdot-m ; \sigma^{2}\right)=\sigma^{2} n H_{n-1}\left(\cdot-m ; \sigma^{2}\right)$. (c) follows from (a) and the fact that $U A^{*} U^{*} H_{n}\left(\cdot-m ; \sigma^{2}\right)=H_{n+1}\left(\cdot-m ; \sigma^{2}\right)$. (d) follows from (a) and Equation 4.1).

Proposition 4.2. For the Poisson measure $\mu_{p}$ on $\mathbb{R}$ with parameter $a$, the following equalities hold:

(a) $S_{\mu_{p}} C_{n}(\cdot ; a)=z^{n}$,

(b) $S_{\mu_{p}} U A U^{*} C_{n}(\cdot ; a)=a n z^{n-1}$,

(c) $S_{\mu_{p}} U A^{*} U^{*} C_{n}(\cdot ; a)=z^{n+1}$,

(d) $S_{\mu_{p}}\left(x C_{n}(\cdot ; a)\right)=z^{n+1}+(n+a) z^{n}+a n z^{n-1}$.

Proof. The idea is similar to the proof of Proposition 4.1 with the Hermite polynomials being replaced by the Charlier polynomials.

We point out that for the Poisson case, we have the equalities for operators acting on $\mathcal{H}_{\lambda}$

$$
\widetilde{\alpha}_{N}=\frac{1}{a} \widetilde{A}^{*} \widetilde{A}+a=\widetilde{N}+a
$$

and

$$
\widetilde{A}^{*}+\widetilde{A}+\widetilde{\alpha}_{N}=\left(\frac{1}{\sqrt{a}} \widetilde{A}^{*}+\sqrt{a}\right)\left(\frac{1}{\sqrt{a}} \widetilde{A}+\sqrt{a}\right)
$$

which correspond to the Equations (4.4) and (4.5), respectively.

Then we can apply Propositions 4.1 and 4.2 to get the next result.

Corollary 4.3. Let $\mu_{g}$ be the Gaussian measure on $\mathbb{R}$ with mean $m$ and variance $\sigma^{2}$. Let $\mu_{p}$ be the Poisson measure on $\mathbb{R}$ with the parameter a. Then 
(1) $S_{\mu_{g}}\left((x-m) H_{n}\left(\cdot-m ; \sigma^{2}\right)\right)=\left(\widetilde{A}^{*}+\widetilde{A}\right) S_{\mu_{g}} H_{n}\left(\cdot-m ; \sigma^{2}\right)$.

(2) $S_{\mu_{p}}\left(x C_{n}(\cdot ; a)\right)=\left(\widetilde{A}^{*}+\widetilde{A}+\widetilde{\alpha}_{N}\right) S_{\mu_{p}} C_{n}(\cdot ; a)$.

Now, suppose $\mu$ is a probability measure on $\mathbb{R}$ with finite moments of all orders. Let $\lambda$ be the sequence associated with $\mu$ as given in Equation (2.2). With this $\lambda$, we have a Hilbert space $\mathcal{H}_{\lambda}$ of analytic functions on $\Omega_{\lambda}$ in Equation (3.4) with norm $\|\cdot\|_{\mathcal{H}_{\lambda}}$ in Equation (3.5). Consider the following

Question. Does there exist a unique measure $\widetilde{\mu}$ on $\Omega_{\lambda}$ such that $F \in \mathcal{H}_{\lambda}$ if and only if $F$ is analytic on $\Omega_{\lambda}$ and $F \in L^{2}\left(\Omega_{\lambda}, \widetilde{\mu}\right)$ with

$$
\|F\|_{\mathcal{H}_{\lambda}}^{2}=\int_{\Omega_{\lambda}}|F(z)|^{2} d \widetilde{\mu}(z) ?
$$

Bargmann 9 considered the equality in Equation (4.6) for the multidimensional standard Gaussian case. See also the paper by Gross and Malliavin [13].

Our main results answer the above question for Gaussian and Poisson measures. For convenience, let $\mathcal{H} L^{2}\left(\Omega_{\lambda}, \widetilde{\mu}\right)$ denote the Hilbert space of analytic functions $F$ on $\Omega_{\lambda}$ which are square integrable with respect to $\widetilde{\mu}$. The norm on $\mathcal{H} L^{2}\left(\Omega_{\lambda}, \widetilde{\mu}\right)$ is the $L^{2}(\widetilde{\mu})$-norm.

To answer the above question, we consider a criterion to check whether a measure $\nu$ satisfying the equation

$$
\int_{\mathbb{C}} \bar{z}^{m} z^{n} d \nu(z)=\gamma_{m, n}
$$

is unique for given moments $\left\{\gamma_{m, n}\right\}$.

Proposition 4.4. Suppose $\left\{\gamma_{m, n}\right\}$ satsifies the condition

$$
\lim _{n \rightarrow \infty} \frac{\gamma_{n, n}^{1 / n}}{n^{2}}=0 .
$$

Then the measure $\nu$ satisfying Equation (4.7) is unique.

Proof. Apply the Schwartz inequality to get

$$
\left|\gamma_{m, n}\right|=\left|\int_{\mathbb{C}} \bar{z}^{m} z^{n} d \nu(z)\right| \leq\left(\gamma_{m, m} \gamma_{n, n}\right)^{\frac{1}{2}} .
$$

Therefore the function

$$
g(t, s)=\sum_{m, n=0}^{\infty} \gamma_{m, n} \frac{t^{m} s^{n}}{m ! n !}
$$

converges absolutely for $t, s \in \mathbb{C}$ by Equation (4.8). In fact, we see that

$$
\begin{aligned}
\int_{\mathbb{C}} \sum_{m, n=0}^{\infty} \frac{\left|(t \bar{z})^{m}(s z)^{n}\right|}{m ! n !} d \nu(z) & \leq \sum_{m, n=0}^{\infty} \frac{\left(\gamma_{m, m} \gamma_{n, n}\right)^{\frac{1}{2}}}{m ! n !}|t|^{m}|s|^{n} \\
& \leq\left(\sum_{n=0}^{\infty} \frac{\gamma_{n, n}^{\frac{1}{2}}}{n !} R^{n}\right)^{2}<\infty
\end{aligned}
$$

for $t, s \in \mathbb{C},|t|,|s| \leq R$. Equation (4.9) implies that $\exp [t \bar{z}+s z]$ is integrable with respect to a measure $\nu$ and

$$
\int_{\mathbb{C}} \exp [t \bar{z}+s z] d \nu(z)=g(t, s)
$$


holds. Therefore the characteristic function of a measure $d \nu(x, y)=d \nu(z), z=$ $x+i y$, satisfies the equality

$$
\int_{\mathbb{R}^{2}} \exp [i \xi x+i \eta y] d \nu(x, y)=g\left(\frac{i \xi+\eta}{2}, \frac{i \xi-\eta}{2}\right)
$$

for any $\xi, \eta \in \mathbb{R}$. Hence $\nu$ is unique.

Remark 4.5. We are unable to find any literature dealing with the moment problem for measures on the complex plane $\mathbb{C}$. The above proposition gives a sufficient condition for the uniqueness of a measure on $\mathbb{C}$ in the moment problem. On the other hand, many authors have studied the moment problem for measures on $\mathbb{R}$.

Theorem 4.6. Let $\mu_{g}$ be the Gaussian measure with mean $m$ and variance $\sigma^{2}$. Let $\mathcal{H}_{\lambda}$ be the Hilbert space associated with $\mu_{g}$ as in Equation (3.4), i.e., $\Omega_{\lambda}=\mathbb{C}$ and $\lambda_{n}=\sigma^{2 n} n$ !. Then there exists a unique measure $\widetilde{\mu}$ on $\mathbb{C}$ such that $\mathcal{H}_{\lambda}=\mathcal{H} L^{2}(\mathbb{C}, \widetilde{\mu})$ and Equation (4.6) holds.

Proof. Consider the above question for the Gaussian measure $\mu_{g}$ with mean $m$ and variance $\sigma^{2}$. In this case, we have $\Omega_{\lambda}=\mathbb{C}$ by Equation (4.3) and $\lambda_{n}=\sigma^{2 n} n$ ! by Equation (4.2).

The uniqueness of $\widetilde{\mu}$ follows from Proposition 4.4. Thus we only need to find a measure $\widetilde{\mu}$ on $\mathbb{C}$ such that

$$
\int_{\mathbb{C}} \bar{z}^{m} z^{n} d \widetilde{\mu}(z)=\delta_{m, n} \sigma^{2 n} n !
$$

Suppose $\widetilde{\mu}$ is given by $d \widetilde{\mu}(z)=\frac{1}{2 \pi} d \rho(r) d \theta$ for $z=r e^{i \theta}$. Then Equation (4.10) becomes

$$
\frac{1}{2 \pi} \int_{0}^{\infty}\left(\int_{0}^{2 \pi} e^{-i(m-n) \theta} d \theta\right) r^{m+n} d \rho(r)=\delta_{m, n} \sigma^{2 n} n !
$$

This equality is obviously valid when $m \neq n$. Thus we are looking for $\rho$ such that

$$
\int_{0}^{\infty} r^{2 n} d \rho(r)=\sigma^{2 n} n !
$$

But it is easy to see that $\rho$ is given by

$$
d \rho(r)=\frac{2}{\sigma^{2}} r \exp \left[-\frac{r^{2}}{\sigma^{2}}\right] d r
$$

The resulting measure

$$
d \widetilde{\mu}_{g}(z)=\frac{1}{\pi \sigma^{2}} r \exp \left[-\frac{r^{2}}{\sigma^{2}}\right] d r d \theta, \quad z=r e^{i \theta},
$$

is a Gaussian measure on $\mathbb{C}$. Hence we have proved the existence of a measure $\widetilde{\mu}$ satisfying Equation (4.6).

Remark 4.7. The measure given in Equation (4.12) is the unique measure satisfying Equation (4.11) since the sequence $\lambda_{n}=\sigma^{2 n} n$ ! satisfies the condition

$$
\sum_{n=1}^{\infty}\left(\lambda_{n}\right)^{-\frac{1}{2 n}}=\infty
$$

in Theorem 1.11 of the book by Shohat and Tamarkin [24]. However, in general it is not true that a measure $d \widetilde{\mu}(z)$ on $\mathbb{C}$ can be written as $\frac{1}{2 \pi} d \rho(r) d \theta$. Thus we really need Proposition 4.4 to show the uniqueness of $\widetilde{\mu}$. 
Observe that we can also apply the above arguments to the Poisson measure $\mu_{p}$. Thus we have the corresponding theorem for $\mu_{p}$.

Theorem 4.8. Let $\mu_{p}$ be the Poisson measure with parameter a. Let $\mathcal{H}_{\lambda}$ be the Hilbert space associated with $\mu_{p}$ as in Equation (3.4), i.e., $\Omega_{\lambda}=\mathbb{C}$ and $\lambda_{n}=a^{n} n$ !. Then there exists a unique measure $\widetilde{\mu}$ on $\mathbb{C}$ such that $\mathcal{H}_{\lambda}=\mathcal{H} L^{2}(\mathbb{C}, \widetilde{\mu})$ and Equation (4.6) holds.

In fact, it is easy to see that the unique measure $\widetilde{\mu}$ for $\mu_{p}$ is also a Gaussian measure on $\mathbb{C}$ with $a$ replacing $\sigma^{2}$ in Equation (4.12). In particular, we see that when $a=\sigma^{2}$ (i.e., $\mu_{g}$ and $\mu_{p}$ have the same variance) the Segal-Bargmann representing spaces for $\mu_{g}$ and $\mu_{p}$ are the same space, namely, the space $\mathcal{H} L^{2}\left(\mathbb{C}, \widetilde{\mu}_{g}\right)$. However, the multiplication operators for $\mu_{g}$ and $\mu_{p}$ are decomposed quite differently on $\mathcal{H} L^{2}\left(\mathbb{C}, \widetilde{\mu}_{g}\right)$.

Remark 4.9. Hudson-Parthasarathy 14] and Ito-Kubo 15 obtained similar results presented in this section from the viewpoint of quantum stochastic calculus and white noise calculus (cf. 19]), respectively. On the relationship between infinite dimensional Gaussian analysis and the Segal-Bargmann transform, see the papers by Asai-Kubo-Kuo [5] 6, 7, 8], Cochran-Kuo-Sengupta 12], Kubo-Yokoi [18, Lee 20], Segal 22, 23, Yokoi [26] and the references therein.

Remark 4.10. Recently, the $q$-deformed versions of Theorems 4.6 and 4.8 have been discussed by the first author [4] following the technique in [3]. We remark that the case of $q$-deformed Gaussian measure has been investigated earlier by van LeeuwenMaassen 21. However, their method of analysis is slightly different from that in [3, 4]. In addition, Krölak examined the Segal-Bargmann space associated with the $q$-commutation relation for $q>1$.

\section{ACKNOWLEDGMENTS}

The authors would like to thank the referee for pointing out several relevant papers. The results in this paper were obtained during several visits of Asai and Kuo to Hiroshima University. Asai and Kuo want to express their deep appreciation to Professor I. Kubo for his invitation and kindness during their visits. They are thankful to the Mathematics Department of Hiroshima University for the warm hospitality. Kuo is grateful for the financial support of Monbu-Kagaku-Sho (Ministry of Education and Science) during his visit May 20-August 19, 2001.

\section{REFERENCES}

1. L. Accardi and M. Bożejko, Interacting Fock space and Gaussianization of probability measures. Infinite Dimensional Analysis, Quantum Probability and Related Topics, 1 (1998), 663-670. MR 2000d:60158

2. L. Accardi, Y.-G. Lu, and I. Volovich, The QED Hilbert module and interacting Fock spaces. IIAS reports 1997-008, Pub. of IIAS (Kyoto), 1997.

3. N. Asai, Analytic characterization of one-mode interacting Fock space. Infinite Dimensional Analysis, Quantum Probability and Related Topics, 4 (2001), 409-415.

4. N. Asai, Integral transform and Segal-Bargmann representation associated to q-Charlier polynomials. Preprint (2001), http://arXiv.org/abs/math.CA/0104260; to appear in Quantum Information IV (T. Hida and K. Saitô, eds.).

5. N. Asai, I. Kubo, and H.-H. Kuo, Bell numbers, log-concavity, and log-convexity. Acta Appl. Math., 63 (2000), 79-87. MR 2002f:60130

6. N. Asai, I. Kubo, and H.-H. Kuo, CKS-space in terms of growth functions. in: Quantum Information II, T. Hida and K. Saitô (eds.) World Scientific, 2000, pp. 17-27. 
7. N. Asai, I. Kubo, and H.-H. Kuo, Roles of log-concavity, log-convexity, and growth order in white noise analysis. Infinite Dimensional Analysis, Quantum Probability, and Related Topics, 4 (2001) 59-84. MR 2002a:46055

8. N. Asai, I. Kubo, and H.-H. Kuo, General characterization theorems and intrinsic topologies in white noise analysis. Hiroshima Math. J., 31 (2001), 299-330. MR 2002f:60131

9. V. Bargmann, On a Hilbert space of analytic functions and an associated integral transform, I. Comm. Pure Appl. Math., 14 (1961), 187-214. MR 28:486

10. V. Bargmann, On a Hilbert space of analytic functions and an associated integral transform, II. Comm. Pure Appl. Math., 20 (1967), 1-101. MR 34:1836

11. T. S. Chihara, An Introduction to Orthogonal Polynomials. Gordon and Breach, 1978. MR 58:1979

12. W. G. Cochran, H.-H. Kuo, and A. Sengupta, A new class of white noise generalized functions. Infinite Dimensional Analysis, Quantum Probability and Related Topics, 1 (1998), 43-67. MR 99d:46062

13. L. Gross and P. Malliavin, Hall's transform and the Segal-Bargmann map. in: Itô Stochastic Calculus and Probability Theory, N. Ikeda et al. (eds.) Springer-Verlag, 1996, pp. 73-116. MR 98j:22010

14. R. L. Hudson and K. R. Parthasarathy, Quantum Ito's formula and stochastic evolutions. Comm. Math. Phys., 93 (1984), 301-323. MR 86e:46057

15. Y. Ito and I. Kubo, Calculus on Gaussian and Poisson white noises. Nagoya Math. J., 111 (1988), 41-84. MR 90a:60118

16. I. Krölak, Measures connected with Bargmann's representation of the q-commutation relation for $q>1$. Banach Center Publ., 43 (1998), 253-257. MR 99h:81095

17. I. Kubo and H.-H. Kuo, Finite dimensional Hida distributions. J. Funct. Anal., 128 (1995), 1-47. MR 96f:60070

18. I. Kubo and Y. Yokoi, Generalized functions and fluctuations in fluctuation analysis. in: Mathematical Approach to Fluctuations, Vol.II, T. Hida et al. (eds.) World Scientific, 1993, pp. 203-230.

19. H.-H. Kuo, White Noise Distribution Theory. CRC Press, 1996. MR 97m:60056

20. Y.-J. Lee, Analytic version of test functionals, Fourier transform and a characterization of measures in white noise calculus. J. Funct. Anal., 100 (1991), 359-380. MR 92k:60186

21. H. van Leeuwen and H. Maassen, A q deformation of the Gauss distribution. J. Math. Phys., 36 (1995), 4743-4756. MR 97a:81104

22. I. E. Segal, Mathematical characterization of the physical vacuum for a linear Bose-Einstein field. Illinois J. Math., 6 (1962), 500-523. MR 26:1075

23. I. E. Segal, The complex wave representation of the free Boson field. in: Essays Dedicated to M. G. Krein on the Occassion of His 70th Birthday, Advances in Math.: Supplementary Studies Vol.3, I. Goldberg and M. Kac (eds.) Academic, 1978, pp. 321-344. MR 82d:81069

24. J. Shohat and J. Tamarkin, The Problem of Moments. Math Surveys 1, Amer. Math. Soc., 1943. MR 5:5c

25. M. Szegö, Orthogonal Polynomials. Coll. Publ. 23, Amer. Math. Soc., 1975. MR 51:8724

26. Y. Yokoi, Simple setting for white noise calculus using Bargmann space and Gauss transform. Hiroshima Math. J., 25 (1995), 97-121. MR 96h:46060

International Institute for Advanced Studies, Kizu, Kyoto, 619-0225, Japan

Current address: Research Institute for Mathematical Sciences, Kyoto University, Kyoto, 6068502, Japan

E-mail address: asai@kurims.kyoto-u.ac.jp

Department of Mathematics, Graduate School of Science, Hiroshima University, Higashi-Hiroshima, 739-8526, JAPAN

E-mail address: kubo@math.sci.hiroshima-u.ac.jp

Department of Mathematics, Louisiana State University, Baton Rouge, Louisiana 70803

E-mail address: kuo@math.lsu.edu 